\title{
The representation of moving 3-D objects in apparent motion perception
}

\author{
Souta Hidaka, Yousuke Kawachi, AND Jiro Gyoba \\ Tohoku University, Sendai, Japan
}

\begin{abstract}
In the present research, we investigated the depth information contained in the representations of apparently moving 3-D objects. By conducting three experiments, we measured the magnitude of representational momentum (RM) as an index of the consistency of an object's representation. Experiment 1A revealed that RM magnitude was greater when shaded, convex, apparently moving objects shifted to a flat circle than when they shifted to a shaded, concave, hemisphere. The difference diminished when the apparently moving objects were concave hemispheres (Experiment 1B). Using luminance-polarized circles, Experiment 2 confirmed that these results were not due to the luminance information of shading. Experiment 3 demonstrated that RM magnitude was greater when convex apparently moving objects shifted to particular blurred convex hemispheres with lowpass filtering than when they shifted to concave hemispheres. These results suggest that the internal object's representation in apparent motion contains incomplete depth information intermediate between that of 2-D and 3-D objects, particularly with regard to convexity information with low-spatial-frequency components.
\end{abstract}

One of the most important functions of the visual systems is the reconstruction of the outer world. For example, blinking or occlusion often results in the discontinuity of retinal inputs. Thus, when we see a moving object, there is a possibility that we perceive discontinuous motion. Nevertheless, in our daily life, we usually perceive the objects in the outer world to be moving smoothly; therefore, it is assumed that the visual systems reconstruct the representation of smoothly moving objects from discontinuous information in the brain. This mechanism is clearly demonstrated by the phenomenon of apparent motion. When objects that are spatially apart from each other are alternately turned on and off at optimal intervals, observers can perceive the motion between these objects (Wertheimer, 1912). Apparent motion is one of the most famous phenomena reflecting the visual reconstruction system for the outer world.

It is known that the visual systems identify a part of the visual inputs that change across time and space as a single object, especially in an ambiguous apparent motion display where there is more than one possible match between the visual inputs. This is referred to as the correspondence problem (Ullman, 1979). It is known that the correspondence-solving process predominantly uses the distance between the apparently moving objects or the degree of proximity between them, relative to shape information (Burt \& Sperling, 1981; Kolers, 1972; Scholl, 2001; Ullman, 1978). Although some studies have suggested that shape information or surface features, such as spatial frequency (Green, 1986; Watson, 1986) and relative phase information (Sekuler \& Bennett, 1996), are used for the correspondence problem, the proximity between objects should be controlled in these cases (Scholl, 2001).

It has also been noted that visual systems complete the internal representations of apparently moving objects in the motion path and that these representations mediate apparent motion perception (Kolers, 1972; Kolers \& von Grünau, 1976; Robins \& Shepard, 1977; Shepard \& Judd, 1976; Shepard \& Zare, 1983). Kolers and von Grünau demonstrated a striking example of this completion process. When a pair of apparently moving objects of different shapes (a triangle and a square) were sequentially presented, these objects appeared to gradually change their shape in the apparent motion path. Moreover, some researchers have confirmed the existence of the internal representation through a variety of methods, such as letter discrimination performance (Yantis \& Nakama, 1998), priming effects on object recognition (Kourtzi \& Shiffrar, 1997, 1999), and attentive tracking (Shioiri, Cavanagh, Miyamoto, \& Yaguchi, 2000). A neurophysiological study has also revealed that the overlapping cortical area of the human motion-processing complex (MT+) and the lateral occipital complex (LOC) become activated when participants observe the apparent motion path (T. Liu, Slotnick, \& Yantis, 2004; see also Zhuo et al., 2003).

\section{Depth Information and \\ Apparent Motion Perception}

Similar to apparent motion perception, the visual systems reconstruct 3-D depth information in the brain because all retinal images are 2-D (Marr, 1982). Many researchers have investigated the relationship between ap-

S. Hidaka, hidaka@sal.tohoku.ac.jp 
parent motion perception and spatial depth information. Ullman (1978) found that the strength of correspondence between the apparently moving objects is determined not on the basis of perceived 3-D distance information, but on the basis of retinal 2-D distance information (see also Mutch, Smith, \& Yonas, 1983). In contrast, some researchers have postulated that apparent motion was perceived with 3-D information (Atteneave \& Block, 1973; Foster, 1975, 1978; Green \& Odom, 1986; Koriat, 1994; Shepard \& Judd, 1976; Tse \& Logothetis, 2002). For example, the trajectory of apparent motion was perceived on the basis of 3-D coordinates in space (Foster, 1975, 1978; Shepard \& Judd, 1976). Similarly, Tse and Logothetis found that the 3-D shapes presented before motion displays biased 3-D motion perception in the transformational apparent motion display in which an object is replaced at some time by another overlapping object. However, the debate on which depth information (2-D or 3-D) plays a dominant role in apparent motion perception remains unresolved. Dawson (1991) suggested that the visual systems separately use 2-D and 3-D depth information depending on the types of processing stages; the correspondence problem is solved with a 2-D coordinate system, whereas motion quality or appearance of trajectories is associated with higher order processing involving 3-D properties. Marr (1982) suggested a similar distinction.

Previous research on apparent motion has focused primarily on information related to the correspondence problem and motion quality or appearance of trajectories. With regard to depth information, research has focused primarily on spatial components. However, although the objects themselves contain depth properties, little research has been conducted on what types of depth information are contained in the internal representations of the objects in apparent motion. Previous research on apparent motion suggests that the visual systems use low-level coarse information. For example, Ramachandran, Ginsburg, and Anstis (1983) proposed that in comparison with shape information, predominantly low-spatial-frequency and texture information are utilized for apparent motion perception. Tse and Logothetis (2002) also suggested that form analysis in the motion path may involve an intermediate representation, particularly at the contour and surface levels. Moreover, from the viewpoint of processing load, the utilization of coarse information appears to be reasonable. Dawson (1991) pointed out that apparent motion perception has limited capacity for an attentional processing load. Hence, coarse information could attenuate the high processing load for object recognition in motion perception. On the basis of these assumptions, it can be considered that the internal representations of moving 3-D objects contain incomplete depth properties in apparent motion perception.

On the basis of this consideration, Hidaka, Kawachi, and Gyoba (2008) investigated the depth information contained in object representation in the apparent motion path. They measured the effect of probe objects briefly presented in the motion path on perceived motion smoothness. The probe objects were flat circles and convex and concave hemispheres defined by shading (Kleffner \& Ramachandran, 1992; Ramachandran, 1988; see Figure 1)

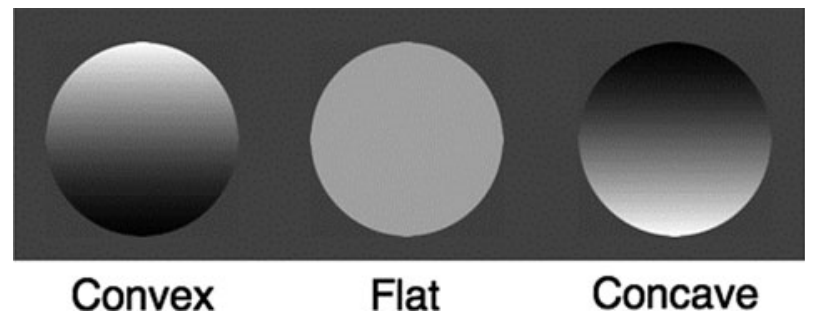

Figure 1. Stimuli used in Experiment 1. The left circle often appears to be a convex hemisphere; the center circle, flat; and the right circle, a concave hemisphere.

or binocular disparity. When the apparently moving objects were convex, the motion was perceived as smoother when the probe object was flat than when it was concave. They also revealed that this effect disappeared when the apparently moving objects were concave. Moreover, these results were consistent between both depth cues. On the basis of these results, they suggested that the internal representations of apparently moving objects in the motion path contain intermediate depth properties between 2-D and 3-D, particularly for convexity.

The findings presented by Hidaka et al. (2008) strongly support the hypothesis that apparently moving $3-\mathrm{D}$ objects contain incomplete or intermediate depth properties. However, in a strict sense, this finding is limited to the internal representation in the midpoint of the apparent motion path. In the present research, we attempted to extend these previous findings to the internal object representations maintained in the entire path of apparent motion. For this purpose, we used the amount of mislocalization of apparently moving objects as an index of the consistency of object identity.

\section{Interaction Between Localizations of Moving Objects and Object Identity}

Previous research has reported the interaction between mislocalizations of moving objects and object shape or identity. Thus, an examination of representational momentum (RM) may be useful for determining what types of depth information are represented in apparently moving objects. RM is a bias in memory wherein the localizations of vanishing points of a moving target are displaced forward in the direction of target motion (Freyd \& Finke, 1984; Hubbard, 1995, 2005), and this bias is influenced by the continuity of object identity. Kelly and Freyd (1987) investigated the effect of the changes in the shape of inducing stimuli in rotating apparent motion. RM occurred when the shapes of the inducing stimuli were consistent or changed smoothly (rectangles becoming thinner or wider) in a rotating motion display. In contrast, radical changes in the shapes of inducing stimuli attenuated RM magnitude. Not only did they study RM for rotation with shape changes, they also obtained similar findings regarding RM for changes in shape itself. These results clearly suggest that the constancy of object shape or identity is critical for the occurrence of RM.

It was also considered that changes that affect RM magnitude are highly related to object perception. Brehaut and 
Tipper (1996) measured RM magnitude for changes in luminance and changes in orientation in the picture plane. In their experiment, they displayed moving targets that rotated horizontally while changing their luminance. They found positive RM for changes in orientation and negative RM for changes in luminance. In contrast, luminance changes had no effect on RM for rotation. However, Munger, Solberg, Horrocks, and Preston (1999) found that luminance changes affected RM for depth rotation of cubes. They obtained RM when cube shading clearly indicated the rotation in depth. In contrast, RM reduction was observed when cubes contained ambiguous shading information or homogeneous luminance information. A comparison of these two findings suggests that luminance changes that are highly related to 3-D object perception have an effect on RM magnitude.

The research on RM indicates that mislocalization of moving objects is stronger when the object's shape or identity is maintained in motion displays. Thus, it could be considered that the mislocalization of moving objects reflects the constancy of shape or identity of moving objects. Moreover, there is considerable evidence that RM is associated with the object recognition process (Hubbard, 1995, 2005; Munger et al., 1999). Therefore, in the present research, we investigated the depth shape information of objects in apparent motion, using RM magnitude as an index of the consistency of apparently moving objects.

\section{Outline of the Present Research}

The purpose of the present research was to investigate the depth properties in the internal representations of apparently moving 3-D objects. Thus, we designed a situation different from that in Kelly and Freyd (1987). In the experiments conducted by Kelly and Freyd, no two inducing objects were identical across the target motion sequence. In our display, the first and third inducing stimuli always possessed the same depth information, and the three inducing stimuli were presented sequentially as they moved horizontally. We compared the RM magnitude for target sequences between the conditions in which the depth shape information of the second inducing stimulus did or did not change. This method enabled us to indirectly investigate the depth information in the internal representations of apparently moving 3-D objects.

With regard to the depth cue, we used shape from shading (Figure 1). It has been confirmed that observers can easily perceive convex or concave 3-D objects (Kleffner \& Ramachandran, 1992; Ramachandran, 1988), depending on the location of the shaded region. On the contrary, figures without shaded regions are perceived to be flat. A shading depth cue enables us to easily manipulate depth shape perception - to 2-D or 3-D (convex or concave) just by changing the shaded regions.

In the three experiments, we used RM magnitude as an index of the consistency of the object's representations in order to test the hypothesis that the internal representations of moving 3-D objects contain depth properties intermediate between 2-D and 3-D in apparent motion perception. In Experiment 1, we used convex, concave, and flat objects as the second inducing stimulus. The first and third inducing stimuli were convex hemispheres in Ex- periment $1 \mathrm{~A}$ and concave hemispheres in Experiment 1B. In Experiments 2A and 2B, we used luminance-polarized circles to confirm that the results of Experiment 1 did not stem from the luminance information of shading. Finally, using low-pass-filtered convex hemispheres as the second inducing stimuli, we explored the possibility that the internal representations of moving 3-D objects dominantly contain low-spatial-frequency information.

\section{EXPERIMENT 1}

Although RM occurs when the shape of inducing stimuli is maintained or changes smoothly, any radical shape change that upsets the maintenance of object identity attenuates RM magnitude. As was mentioned above, we presented three-point apparent motion and compared the RM magnitudes for the target sequences with different types of second inducing stimuli. If the second inducing stimulus induced RM reduction for the target sequence, this stimulus could be assumed to be inconsistent with the internal representations of apparently moving objects. Conversely, no RM reduction for the target sequence would imply that the depth information contained in the second inducing stimulus was highly consistent with that of the internal representation. In Experiment 1A, a convex hemisphere was used as the first and third inducing stimuli.

We also investigated whether the internal representations of apparently moving 3-D objects contain asymmetrical depth properties between convexity and concavity. It is known that the visual systems asymmetrically code convexity and concavity in shape perception. For example, with regard to figure-ground segmentation, the predominant processing of convexity has been reported in edge recognition and symmetry detection (Baylis \& Driver, 1995; Driver \& Baylis, 1995) or part discrimination (Bertamini, 2001). Similar asymmetrical properties have also been reported with regard to shape from shading (Kleffner \& Ramachandran, 1992; B. Liu \& Todd, 2004). Kawabe and Miura (2003) illustrated that a region consisting of convex figures was dominantly perceived as a figure in figure-ground segmentation. An asymmetrical effect between convexity and concavity has also been confirmed in motion displays. Shirai and Yamaguchi (2004) revealed that the impression of motion in depth is stronger for convex hemispheres than for concave ones. They also found that the detection of expanding convex hemispheres is more accurate than that of concave ones in visual search tasks. On the basis of these findings, it can be said that for shape perception, the visual systems prefer convexity in both static and dynamic displays. Thus, we could predict that the asymmetrical effect between convexity and concavity would be observed in depth shape information contained in apparently moving objects. If the internal representations of apparently moving objects had the convexity dominance property, the effect of the second inducing stimuli on RM for the target sequence with the concave first and third inducing stimuli would be attenuated, relative to those with the convex first and third inducing stimuli. In Experiment 1B, we explored this possibility using the concave first and third inducing stimuli. 


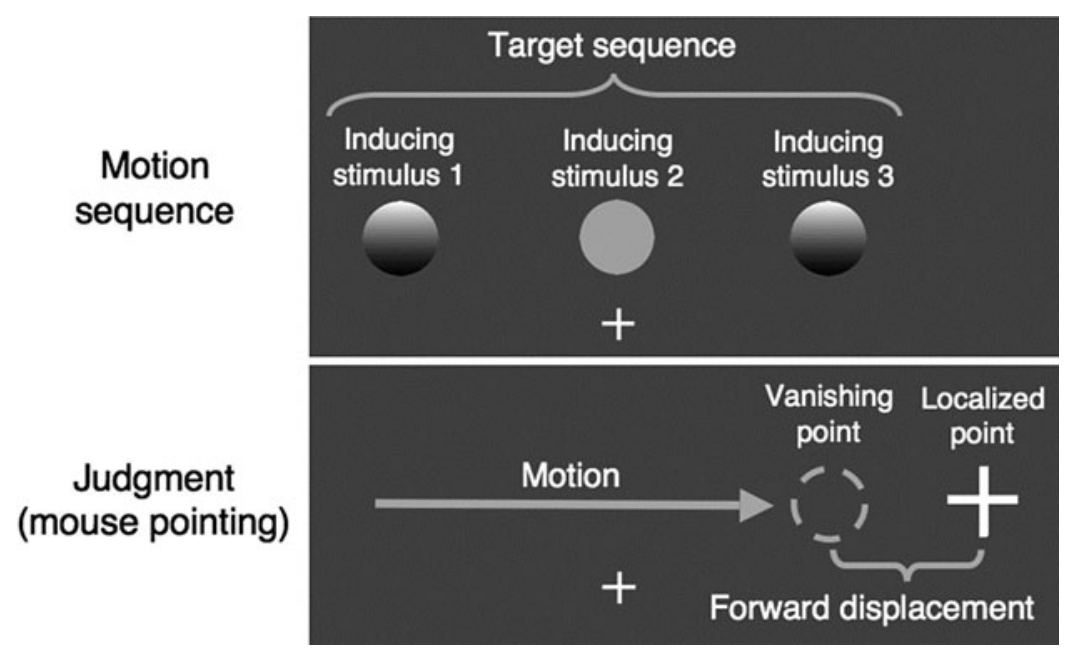

Figure 2. Experimental design for Experiment 1. The mouse cursor was blue and the fixation cross was red in the experiments.

\section{Method}

Participants and Apparatus. This experiment included 8 participants (Tohoku University students): 2 of the authors (S.H. and Y.K.) and 6 participants naive as to the purpose of this experiment. All the participants had normal or corrected-to-normal vision. The stimuli were presented on a CRT display (Sony Trinitron GDMFW900, 24 in.) with a resolution of 1,600 $\times 1,200$ pixels and a refresh rate of $75 \mathrm{~Hz}$. A customized PC (Dell-Dimension 8250) and MATLAB (The MathWorks, Inc.) with the Psychophysics Toolbox (Brainard, 1997; Pelli, 1997) was used to control the experiment. The participants were instructed to place their heads on a chinrest.

Stimuli. We used shaded convex and concave hemispheres $\left(118.6-0 \mathrm{~cd} / \mathrm{m}^{2}\right)$ and a flat circle $\left(59.97 \mathrm{~cd} / \mathrm{m}^{2}\right)$ as inducing stimuli (Figure 1). The luminance value of the flat circle was the averaged luminance of the shaded hemisphere. We used a blue cross $\left(12.36 \mathrm{~cd} / \mathrm{m}^{2}\right)$ as a mouse cursor. These stimuli were presented against a gray background $\left(14.46 \mathrm{~cd} / \mathrm{m}^{2}\right)$. All the stimuli were $0.6^{\circ} \times 0.6^{\circ}$ in visual angle. The horizontal distance between each inducing stimulus was $2.5^{\circ}$. A red fixation cross $\left(0.3^{\circ} \times 0.3^{\circ}, 26.05 \mathrm{~cd} / \mathrm{m}^{2}\right)$ was also presented at the center of the display. Vertical distance between the stimuli and the fixation cross was $0.6^{\circ}$, and the observation distance was $57.3 \mathrm{~cm}$.

Procedure. The experiment was conducted in a dark room. Prior to beginning the experiment, the participants were given instructions and were asked to confirm whether they could perceive the shaded hemispheres (convex or concave) with depth and the flat objects without any depth. All the participants answered in the affirmative. In the target motion sequence, the three inducing stimuli were presented in order (Figure 2). The duration of each inducing stimulus was $150 \mathrm{msec}$. The interstimulus interval (ISI) was $100 \mathrm{msec}$. The first and third inducing stimuli were convex hemispheres in Experiment 1A, whereas they were concave hemispheres in Experiment $1 \mathrm{~B}$. The second inducing stimuli were the convex hemispheres, concave hemispheres, or flat circles. In each trial, one of the second inducing stimuli was presented. It has been reported that RM magnitude for leftward motion is greater than that for rightward motion (Halpern \& Kelly, 1993). In order to investigate the effect of motion direction on RM, the directions of the target sequence (left or right) were randomly intermixed in each trial and counterbalanced in each second inducing stimulus. We set the start positions of the first inducing stimuli as $-3.3^{\circ},-2.8^{\circ},-2.5^{\circ},-2.2^{\circ}$, and $-1.9^{\circ}$ from the fixation point in the rightward motion condition and $1.9^{\circ}, 2.2^{\circ}, 2.5^{\circ}$, $2.8^{\circ}$, and $3.3^{\circ}$ from the fixation point in the leftward motion condition. These starting positions were also randomly intermixed in each trial and counterbalanced in each second inducing stimulus.
After pressing the start button, the participants observed the target sequence wherein the three inducing stimuli appeared to move horizontally. The mouse cursor appeared in the display $100 \mathrm{msec}$ after the target sequence had finished. We asked the participants to use the mouse to localize the cursor at the vanishing point of the third inducing stimulus (Hubbard \& Bharucha, 1988). We also asked them to adjust the center of the cursor to indicate the location perceived as the center of the third inducing stimulus. On each trial, the appearance position of the mouse cursor was randomly selected within $\pm 2^{\circ}$ horizontal positions relative to the fixation point. During the trial, the participants fixated their eyes on the fixation cross. After 6 practice trials, the participants performed 30 trials, which made up the main part of the experiment. The experiment comprised the following factors: second inducing stimuli $(3) \times$ motion directions $(2$ : left/right $) \times$ starting points $(5)$.

\section{Results and Discussion}

RM magnitude (in degrees) was calculated as the horizontal displacement between the judged vanishing points and the actual vanishing points of the third inducing stimulus. When the first and third inducing stimuli were convex hemispheres (Experiment 1A), the mean RM magnitude for the target sequence with each type of second inducing stimuli was as follows: flat $=0.72^{\circ}$, convex $=0.65^{\circ}$, and concave $=0.55^{\circ}$ (Figure $\left.3 \mathrm{~A}\right)$. Onetailed $t$ tests confirmed that all of these RM magnitudes were significantly greater than zero at the $5 \%$ level [flat, $t(7)=2.92$; convex, $t(7)=2.96$; concave, $t(7)=2.44]$. We conducted a two-way repeated measures ANOVA with the second inducing stimuli $(3) \times$ motion direction (2). This revealed the main effect of the second inducing stimuli $[F(2,14)=4.61, p<.03]$. Ryan's (1960) post hoc comparison showed that the RM magnitude was significantly greater for the target sequence with the flat second inducing stimulus than for those with the concave second inducing stimulus $(p<.03)$. When the first and third inducing stimuli were concave hemispheres (Experiment $1 \mathrm{~B}$ ), the RM magnitude for the target sequence with each type of second inducing stimulus was as follows: flat $=0.65^{\circ}$, convex $=0.525^{\circ}$, and concave $=$ $0.528^{\circ}$ (Figure $3 \mathrm{~B}$ ). One-tailed $t$ tests confirmed that all 


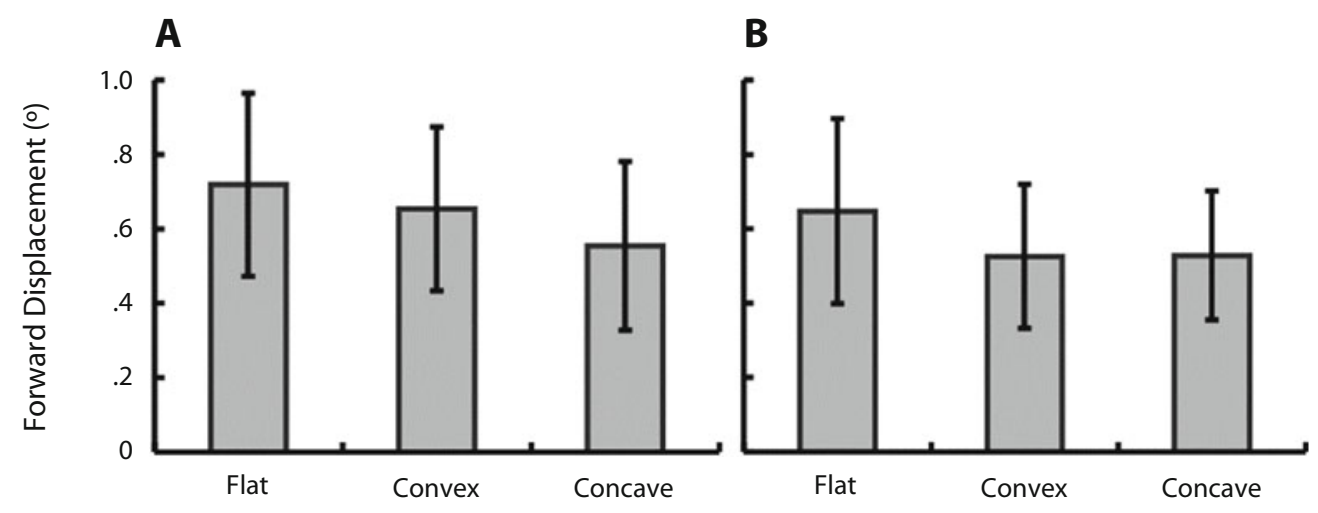

Types of the Second Stimuli

Figure 3. Mean representational momentum magnitude for the target sequence with each second inducing stimulus in Experiment 1. In Experiment 1A, the first and third inducing stimuli were convex hemispheres; in Experiment 1B, concave hemispheres. (A) Results of Experiment 1A. (B) Results of Experiment 1B. The error bars denote mean standard errors $(N=8)$.

of these RM magnitudes were significantly greater than zero at the $5 \%$ level [flat, $t(7)=2.60$; convex, $t(7)=$ 2.71 ; concave, $t(7)=3.04]$. The two-way repeated measures ANOVA revealed no significant effects of the second inducing stimuli $[F(2,14)=1.28, p=.31]$. In both experiments, there were no significant effects of motion direction [Experiment 1A, $F(1,7)=1.69, p=.24$; Experiment $1 \mathrm{~B}, F(1,7)=2.00, p=.20]$.

During the experiments, we observed RM magnitudes that were significantly greater than zero for each target sequence. The results of Experiment 1A revealed that the RM magnitude was significantly greater for the target sequence with the flat second inducing stimulus than for those with the concave second inducing stimulus. This result clearly indicated that shifts from 3-D to 2-D objects did not reduce RM magnitudes. However, the internal representations of apparently moving objects did not appear to contain only 2-D information, because there was no RM reduction for the target sequence with the convex second inducing stimuli. Moreover, as compared with the flat second inducing stimuli, the reduction of RM magnitudes occurred for the target sequence with the concave second inducing stimuli, whose depth information was opposite to that of the first and third inducing stimuli. From these results, we can infer that the internal representations of apparently moving objects contain incomplete or intermediate depth information between 2-D and 3-D properties.

Conversely, we did not obtain any effect of the second inducing stimuli in Experiment 1B. The asymmetrical effects of the second inducing stimuli on RM for the target sequences between the experiments strongly supported our hypothesis that the internal representations predominantly contain the convex depth properties. As was described in the introduction, the asymmetrical properties between convexity and concavity are closely related to shape perception (Baylis \& Driver, 1995; Bertamini, 2001; Kawabe \& Miura, 2003; Kleffner \& Ramachandran, 1992; B. Liu \& Todd, 2004; Shirai \& Yamaguchi, 2004). Therefore, we could infer that the findings obtained in Experiment 1 stemmed from depth shape perception.

The results of Experiment 1 were highly consistent with the findings of our previous research (Hidaka et al., 2008) and suggested that the internal representations of the objects in apparent motion comprise coarse 3-D properties or intermediate depth properties between 2-D and 3-D, particularly with regard to convexity information.

\section{EXPERIMENT 2}

Using a shading depth cue, depth perception could be manipulated easily with changes in the location of the shaded region. This indicated that shading depth cue consisted of luminance polarity information. Luminance profiles consisting of upside-white and downside-black or vice versa play an important role in the perception of convexity or concavity. Thus, it is possible that luminance polarity itself can become the critical cue for object identity, as well as perceived depth information. The results of Experiment 1 revealed that the RM magnitude appears to be relatively greater for the target sequence with the flat second inducing stimulus than for those with the other second inducing stimuli. Whereas the shaded hemispheres contained the luminance polarities, the flat circle consisted of an averaged homogeneous luminance surface. Thus, the surface information of the flat stimulus might be neutral for both the convex and concave hemispheres. With regard to luminance profiles, there exists a possibility that the results of Experiment 1 stemmed not from depth shape information, but from the similarity or consistency of luminance information.

In order to investigate the contribution of luminance property to RM magnitude, we replaced the shaded hemisphere with luminance-polarized circles (Figure 4). It has been confirmed that although these circles have the same luminance polarity as that of shaded hemispheres, observers no longer perceive depth from these circles (Ramachandran, 1988). Thus, using luminance-polarized circles, we 

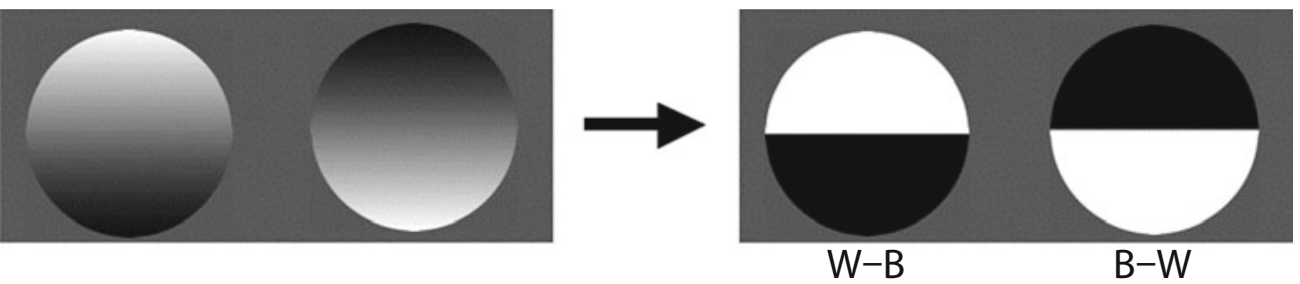

Figure 4. Luminance-polarized circles used in Experiment 2. White-black (W-B) has the same luminance polarity as that of the convex hemispheres. In contrast, the luminance polarity of black-white (B-W) was consistent with that of the concave hemispheres. Note that both induce no depth perception.

could directly investigate the effect of luminance information for RM magnitude. We made the following prediction: If the findings of Experiment 1 stemmed purely from depth shape perception, the results of Experiment 1 would not be replicated in this experiment, in which circles with no depth information were used. In order to confirm this prediction, we conducted an experiment in which the general method of Experiment 1 was replicated, except that luminance-polarized stimuli were used, instead of shaded hemispheres.

\section{Method}

Participants and Apparatus. This experiment included 8 participants (Tohoku University students): 2 of the authors (S.H. and Y.K.) and 6 students who were naive as to the purpose of this experiment, although 3 of them had participated in Experiment 1. All the participants had normal or corrected-to-normal vision. The apparatus was identical to that used in the previous experiment.

Stimuli and Procedure. We used two types of luminancepolarized circles-namely, white-black (W-B) and black-white (B-W) - as stimuli (Figure 4). Whereas W-B had the same luminance profile as that of the convex hemispheres, the luminance polarity of $\mathrm{B}-\mathrm{W}$ was consistent with that of the concave hemispheres. W-B, $\mathrm{B}-\mathrm{W}$, and the flat circles were used as the second inducing stimuli. In Experiment $2 \mathrm{~A}$, we used $\mathrm{W}-\mathrm{B}$ as the first and third inducing stimuli. In contrast, B-W was presented as the first and third inducing stimuli in Experiment 2B. Apart from these differences, the stimulus parameters and procedures were identical to those used in Experiment 1.

\section{Results and Discussion}

As in Experiment 1, we calculated the mean RM magnitude for the target sequence with each type of second inducing stimulus. When the first and third inducing stimuli were $\mathrm{W}-\mathrm{B}$ (Experiment $2 \mathrm{~A}$ ), the mean RM magnitude was as follows: flat $=0.51^{\circ}, \mathrm{W}-\mathrm{B}=0.40^{\circ}$, and $\mathrm{B}-\mathrm{W}=$ $0.42^{\circ}$ (Figure 5A). A one-tailed $t$ test confirmed that the RM magnitude for the target sequence with $\mathrm{W}-\mathrm{B}$ was significantly greater than zero at the $5 \%$ level $[t(7)=2.19]$. The RM magnitudes for the target sequences with the flat and $\mathrm{B}-\mathrm{W}$ second inducing stimuli were marginally significantly greater than zero at the $10 \%$ level [flat, $t(7)=$ 1.69 ; B-W, $t(7)=1.79]$. The two-way repeated measures ANOVA with the second inducing stimuli $\times$ motion direction revealed no significant effects of second inducing stimuli $[F(2,14)=0.65, p=.54]$ and motion direction $[F(1,7)=0.75, p=.42]$. When the first and third inducing stimuli were B-W (Experiment $2 \mathrm{~B}$ ), the mean RM magnitude was as follows: flat $=0.52^{\circ}, \mathrm{W}-\mathrm{B}=0.42^{\circ}$, and $\mathrm{B}-\mathrm{W}=0.45^{\circ}$ (Figure 5B). A one-tailed $t$ test confirmed that all of these RM magnitudes were significantly greater than zero at the $5 \%$ level [flat, $t(7)=2.14 ; \mathrm{W}-\mathrm{B}, t(7)=$ $2.22 ; \mathrm{B}-\mathrm{W}, t(7)=2.57]$. The two-way repeated measures ANOVA revealed no significant effects of second inducing stimuli $[F(2,14)=0.48, p=.63]$ and motion direction $[F(1,7)=1.35, p=.28]$.

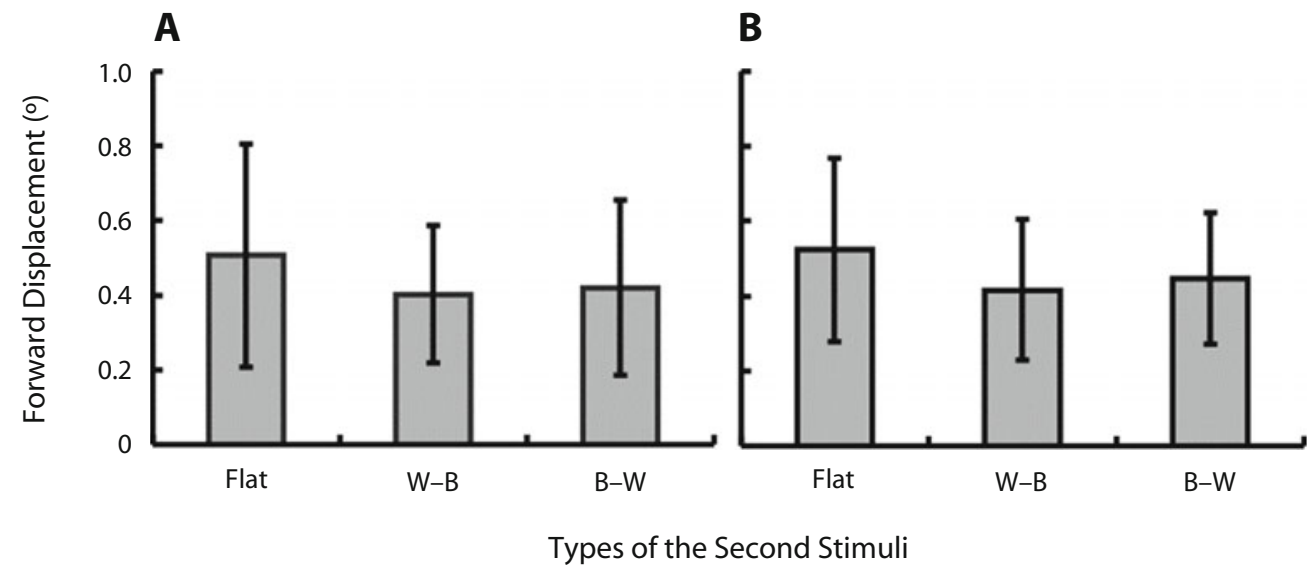

Figure 5. Mean representational momentum magnitude for the target sequence with each second inducing stimulus in Experiment 2. In Experiment $2 A$, the first and third inducing stimulus was white-black (W-B); in Experiment 1B, black-white (B-W). (A) Results of Experiment 2A. (B) Results of Experiment $2 B$. The error bars denote mean standard errors $(N=8)$. 
In Experiment 2A, only the RM magnitude for the target sequence with the $\mathrm{W}-\mathrm{B}$ second inducing stimulus was significantly different from zero. This result suggested that the luminance shift from the $\mathrm{W}-\mathrm{B}$ first and third inducing stimuli to the flat or B-W second inducing stimuli had an attenuating effect on RM. However, this did not appear to be the case, because Experiment 2A also confirmed that there were no significant differences in RM magnitude for the target sequence between each type of second inducing stimulus. Although luminance shifts also occurred in Experiment 2B, all of the RM magnitudes for the target sequences were significantly greater than zero. These results indicated that a reliable amount of RM occurred in this experiment.

Of importance is the fact that the difference in the RM magnitude for the target sequences between the second inducing stimuli disappeared in Experiments $2 \mathrm{~A}$ and $2 \mathrm{~B}$, despite the fact that the luminance profiles of the luminance-polarized circles were identical to those of the shaded hemispheres. Because the results of Experiment 2 did not replicate those of Experiment 1, we confirmed that luminance information was not critical for the results of Experiment 1.

Brehaut and Tipper (1996) measured RM magnitude for changes in orientation and changes in luminance in the picture plane. They found positive RM for changes in orientation and negative RM for changes in luminance. In contrast, luminance changes did not affect RM for rotation in the picture plane. However, Munger et al. (1999) found that luminance changes have an effect on RM for depth rotation of cubes; RM occurred when cube shading clearly indicated depth rotation, and RM reduction occurred when the cubes contained ambiguous shading information or homogeneous luminance information. These findings indicate that luminance information that is highly related to 3-D depth shape perception affects RM magnitude. In line with the previous research, the difference in results between Experiments 1 and 2 clearly suggested that the results of Experiment 1 were obtained on the basis of depth shape perception.

\section{EXPERIMENT 3}

On the basis of the results of Experiment 1A, we assumed that the internal representations of apparently moving objects contain intermediate depth information between 2-D and 3-D image properties. In Experiment 3, we investigated the possibility that the intermediate depth information of internal representations consists of lowspatial-frequency components.

Ramachandran et al. (1983) showed that low spatial frequency is predominantly used in the correspondence problem, as compared with the shape of objects. This appears to be reasonable for the visual systems. Lowspatial-frequency information is globally processed as coarse information, so that it may require a relatively lower processing load than does shape information, which requires detailed processing. Thus, we hypothesized that with regard to depth shape information in apparent motion perception, the visual systems mainly process low-spatialfrequency components. On the basis of this assumption and by using Gaussian low-pass filters, we created four blurred images (B1-B4) of shaded convex hemispheres (Figure 6). It is apparent from these figures that the greater the increase in magnitude of blur, the greater is the decrease in the shading depth perception, although we could still perceive depth from these images. Thus, we could consider these images with low-spatial-frequency dominance to be potent candidates for the internal representations containing intermediate depth properties in apparent motion perception.

In Experiment 3, the general method of Experiment 1A (in which the first and third inducing stimuli were convex hemispheres) was replicated, and the convex, concave, and blurred convex hemispheres were used as the second inducing stimuli. Because the convexity dominance property was confirmed in Experiment 1, we used convex hemispheres as the first and third inducing stimuli and the blurred convex images.

It has been reported that blurred images induce the perceptual forward displacement of moving objects. Fu, Shen, and Dan (2001) found that two blurred vertical stripes that moved horizontally in opposite directions were perceived to be misaligned with each other, although they were actually aligned. They also reported that higher blur intensity induced greater magnitude of misalignment. Thus, there was a possibility that blur itself had the effect of increasing $\mathrm{RM}$. This led to the prediction that RM magnitude would be greater for the target sequences with the blurred second inducing stimuli than for those with the convex and concave second inducing stimuli. It was also predicted that RM magnitudes linearly increase with increasing blur intensity. In contrast, if incomplete depth properties with low-spatial-frequency components were critical for

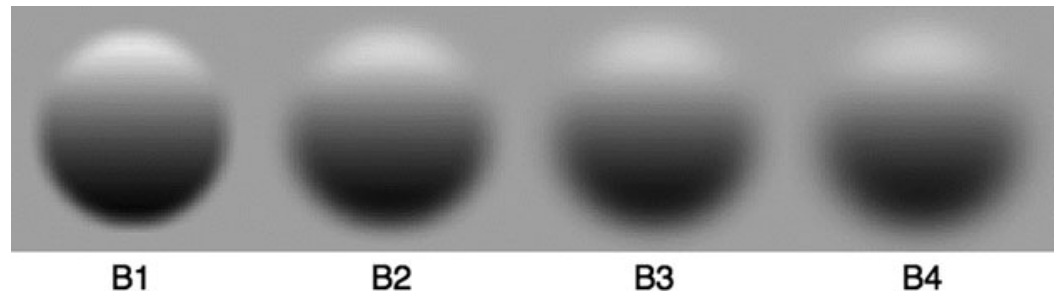

Figure 6. Blurred convex hemispheres used in Experiment 3. The cutoff spatial frequency of the low-pass filter increased from B1 to B4. Note that the blur attenuates the depth perception of convexity. 
RM, the RM magnitude for the target sequences with the blurred second inducing stimuli would be greater than that for the concave second inducing stimulus, whose depth property was opposite to the convex first and third inducing stimuli.

\section{Method}

Participants and Apparatus. This experiment included 8 participants (Tohoku University students): 1 of the authors (S.H.) and 7 students who were naive as to the purpose of the experiment, although 1 of them had participated in Experiment 1. All the participants had normal or corrected-to-normal vision. The apparatus was identical to that used in the previous experiment.

Stimuli and Procedure. In this experiment, the first and third inducing stimuli were convex hemispheres. The second inducing stimuli were convex and concave hemispheres and four types of blurred convex images (B1-B4) created by low-pass filtering (Figure 6). Four low-pass filters were defined by a Gaussian. The $\partial$ of each Gaussian was $\sqrt{6}, \sqrt{ } 12, \sqrt{ } 18$, and $\sqrt{ } 24$ pixels. The convex hemisphere was 64 pixels. The cutoff spatial frequency of each filter was 7, 5, 4, and 3.5 cycles/pixel. It was necessary to keep a consistent luminance contrast between the unblurred and blurred images so that the gray background was $59.97 \mathrm{~cd} / \mathrm{m}^{2}$, which was the averaged luminance value of the shaded hemispheres. We set the size of the stimuli as twice that of the previous experiments for the clear presentation of the blurred images. In order to equate the visual angles of the stimuli between the experiments, we set the observation distance as $114.6 \mathrm{~cm}$ in this experiment. After 6 practice trials, the observers participated in 60 trials, which composed the main part of the experiment. It comprised the second inducing stimuli $(6) \times$ motion directions $(2$ : left/right $) \times$ starting points (5). Apart from these differences, the stimulus parameters and procedures were identical to those used in Experiments 1 and 2.

\section{Results and Discussion}

As in Experiments 1 and 2, we calculated the mean RM magnitude for the target sequence with each type of second inducing stimulus. The mean RM magnitude was as follows: convex $=0.49^{\circ}, \mathrm{B} 1=0.48^{\circ}, \mathrm{B} 2=0.54^{\circ}, \mathrm{B} 3=$ $0.53^{\circ}, \mathrm{B} 4=0.49^{\circ}$, and concave $=0.27^{\circ}$ (see Figure 7). A one-tailed $t$ test confirmed that, except for the concave second inducing stimulus $[t(7)=1.26, p=.13]$, all of these RM magnitudes were significantly greater than zero at the $5 \%$ level [convex, $t(7)=2.36 ; \mathrm{B} 1, t(7)=1.89 ; \mathrm{B} 2$, $t(7)=2.37$; B3, $t(7)=2.15$; B4, $t(7)=2.08$ ]. A twoway repeated measures ANOVA with the second inducing stimuli $\times$ motion direction revealed significant effects of the second inducing stimuli $[F(5,35)=3.08, p<.03]$. Ryan's (1960) post hoc comparison showed that the RM magnitudes were significantly greater for the target sequences with B2 and B3 than for those with the concave object $(p<.03)$. In contrast, there were no significant differences in motion direction $[F(1,7)=1.98, p=.20]$.

In this experiment, all RM magnitudes were significantly greater than zero, except for the RM magnitude for the target sequence with the concave second inducing stimulus. The results revealed that the RM magnitudes were greater for the target sequences with the specific blurred images (B2 and B3) than for those with the concave second inducing stimulus, whose depth information was opposite to that of the convex first and third inducing stimuli. We also confirmed that the RM magnitudes for the target sequences with the blurred images did not linearly increase. These results were not consistent with the explanation based on the effect of blur itself. The findings in the present experiment strongly support our hypothesis that apparently moving objects would be represented as low-spatial-frequency-dominant images with incomplete depth information. As was mentioned above, depth shape perception appeared to be different among the blurred images. Thus, it might also be inferred that the depth information of the particular blur images was highly consistent with that of the internal representations of apparently moving 3-D objects.

Because the visual systems have a limited capacity of temporal resolution, moving objects can be perceived as blurred images (Burr, 1980). Nevertheless, at the perceptual level, humans can perceive moving objects as sharp images. Some researchers have noted that the visual systems have a mechanism that sharpens the moving blurred images into clear images in the brain (Bex, Edgar, \& Smith, 1995; Ramachandran, Rao, \& Vidyasagar, 1974). It is assumed that deblurring or motion sharpening is completed by the spatiotemporal integration or summation of the blurred images (Burr, 1980; Burr \& Ross, 1986). In Experiment 3, it was suggested that the blurred convex second inducing stimuli would be consistent with the internal representations of apparently moving 3-D objects.

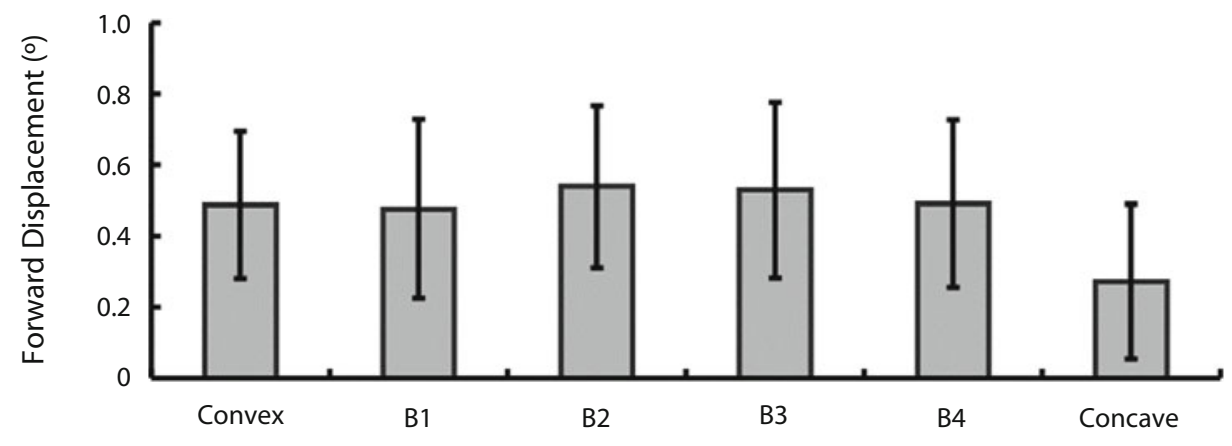

Types of the Second Stimuli

Figure 7. Mean representational momentum magnitude for the target sequence with each second inducing stimulus in Experiment 3. The error bars denote mean standard errors $(N=8)$. 
Thus, we assumed that the present research regarding the internal representations would shed light on the intermediate processing stage, wherein the visual systems are in the process of establishing the perception of moving objects.

\section{GENERAL DISCUSSION}

In all three experiments, we investigated the depth information contained in apparently moving 3-D objects. It has been assumed that the mislocalization of moving objects reflects the consistency of object shape or identity in motion perception (Kelly \& Freyd, 1987). Therefore, we measured RM magnitudes in cases in which the depth shape information of the second inducing stimulus did or did not change from that of the first and third inducing stimuli in the three-point apparent motion display.

When the first and third inducing stimuli were convex, the results showed no reduction of RM for the target sequence with shifts from 3-D to 2-D objects (flat) and showed RM reduction for the target sequence with shifts to opposite depth shape (concave) information (Experiment 1A). On the basis of these findings, we concluded that the internal representations of the objects in apparent motion contain coarse or intermediate depth properties between 2-D and 3-D images. The effect of the second inducing stimuli diminished when the first and third inducing stimuli were concave hemispheres (Experiment 1B). The asymmetrical effect of the second inducing stimuli between the experiments suggested that convexity information is encoded more strongly or more quickly in the internal representations.

In Experiment 2, we replicated the general method of Experiment 1, except that luminance-polarized stimuli were used instead of shaded hemispheres. Although the luminance polarity of these circles was consistent with that of the shaded hemispheres, we no longer perceived depth from these circles (Ramachandran, 1988). Because the results of Experiment 2 did not replicate those of Experiment 1, we confirmed that the findings of Experiment 1 resulted not from luminance information but from an object's depth information.

Finally, in Experiment 3, we explored the possibility that the internal representations of apparently moving 3-D objects contain dominantly low-spatial-frequency properties. When the convex first and third inducing stimuli shifted to the particular blurred images ( $4-5$ cycle/pixel cutoff frequencies), the RM magnitude for the target sequence was significantly greater than that for those with the concave second inducing stimulus. Thus, these results suggest that the internal representations are low-spatial-frequencydominant images with coarse depth information.

The following two aspects clearly indicate that the findings of the present research stem from depth shape perception. The first is the dominance of convex representations. It is known that visual systems prefer information regarding convexity, relative to concavity, in shape perception (Baylis \& Driver, 1995; Driver \& Baylis, 1995), shape from shading (Kawabe \& Miura, 2003; B. Liu \& Todd, 2004), and motion in depth (Shirai \& Yamaguchi, 2004). This asymmetrical effect of convexity was also observed in the present research. In Experiment 1, the difference of the second inducing stimuli with regard to the magnitude of RM for the target sequence was obtained only when the first and third inducing stimuli were convex hemispheres. The second aspect is the importance of shading information. When the shaded hemispheres were replaced with luminance-polarized circles, the effects of the second inducing stimuli on RM for the target sequence diminished. In line with previous research (Brehaut \& Tipper, 1996; Munger et al., 1999), luminance information that is highly associated with 3-D depth shape perception affected the RM magnitude for the target sequence.

The assumption that our findings are actually related to depth shape information can be further confirmed by the results of Experiment 3. Fu et al. (2001) reported that blurred images induce perceptual forward displacement of moving objects and that the amount of the displacement increases linearly with increasing blur intensity. The present research showed the significant increments of RM magnitudes for the target sequences with the particular blurred second inducing stimuli, in comparison with those with the concave second inducing stimuli; on the other hand, the RM magnitude for the target sequences with the blurred second inducing stimuli did not increase linearly. These results indicated that our results could not be explained simply by the effect of blur itself.

Thompson, Brooks, and Hammett (2006) reported that the perceived speed of moving objects became faster in lower contrast conditions. It was also reported that RM become larger with faster velocities (Freyd \& Finke, 1985; but see also Munger \& Minchew, 2002; Munger \& Owens, 2004). Thus, in Experiment 3, there existed the possibility that low saliency induced by blur might have increased the perceived speed of apparently moving objects, thereby increasing the resulting RM magnitude. Although this idea also predicts linear increments of RM magnitudes for the target sequences from B1 to B4, this was not the case in the present experiment. The results of Experiment 3 are also incompatible with the saliency-based interpretation.

The present findings may suggest that the spatial frequency components contained in blurred images are optimal for the occurrence of RM. If the spatial frequency components of the blurred images were critical to the result of Experiment 3, the RM magnitude should have been significantly higher for the target sequences with the blurred images than for those with the convex and the concave second inducing stimuli, because the spatial frequency components of both stimuli were consistent. However, we did not confirm such a tendency. Only the RM magnitudes for the target sequences with the particular blurred images were significantly higher than those for the target sequences with the concave second inducing stimulus, whose depth information was opposite to the convex first and third inducing stimuli. The interpretation based only on the spatial frequency components could not fully explain our results. In line with the findings of Experiments 1 and 2, we suppose that not only the spatial frequency components, but also the incomplete depth shape information contained in the blurred images was critical for the results of Experiment 3. 
The present research used RM magnitude as an index of the consistency of the object's internal representation, because it has been considered that RM magnitude reflects the consistency of object identity in an apparent motion sequence (Kelly \& Freyd, 1987). Kelly and Freyd reported $\mathrm{RM}$ reduction for rotation when a rectangle changed its shape to a square. In contrast, there was no RM reduction when rotating rectangles gradually changed their size (thinner or wider). Because luminance-level changes commonly occur in both cases, it can be assumed that the object-level change has an effect on RM. The results of Experiment 2 support this idea, because the luminance information itself did not affect RM magnitude (see also Brehaut \& Tipper, 1996; Munger et al., 1999). Discontinuity of object identity would make an apparent motion display appear to be discrete presentations of multiple objects, rather than the continuous motion of a single object. As a result, RM reduction would occur. Recent research on visible persistence supports this idea. Moore, Mordkoff, and Enns (2007) presented a circular moving object that radically changed its size one frame before the final frame. They found that observers perceived two moving objects in the final frame even though the frame actually contained one object. They also confirmed that objectlevel change, and not physical-level change, triggered this phenomenon. Since they called this phenomenon objectmediated updating, it could be considered that the discontinuity of object identity in motion displays induces the perception of multiple objects.

All these views suggest the involvement of objectlevel processing in the occurrence of RM. In this study, RM magnitude for the target sequence was greater when the convex first and third inducing stimuli shifted to the flat second inducing stimulus than when they shifted to the concave ones (Experiment 1A). Conversely, although the convex second inducing stimulus was identical to the first and third inducing stimuli moving objects, it did not significantly increase the RM magnitude, relative to those with the concave second inducing stimuli. These counterintuitive results suggest that measurement of RM magnitude taps the processing of object representation. This idea is also supported by the results of Experiment 3. The obtained RM magnitude may correspond to the representational stage that sharpens the blurred moving images to sharp images in the brain (Bex et al., 1995; Burr, 1980; Ramachandran et al., 1974). The results from this study indicate that RM magnitude can be a new index highlighting the process of representational level for a visual object.

Like the findings obtained in our previous research (Hidaka et al., 2008), the results in this study suggest that the internal representations of moving 3-D objects across the entire trajectory of an object undergoing apparent motion contain coarse depth information intermediate between 2-D and 3-D depth information, particularly for convexity information. Our present findings appear to be highly consistent with the concept of an intermediate representation stage (2.5-D sketch) suggested by Marr (1982). This stage mediates the transformation of 2-D retinal images into 3-D representations by the estimation of surface direction, on the basis of the predominant processing of con- vexity. Moreover, Tse and Logothetis (2002) suggested that form analysis in apparent motion is completed by an intermediate representation, particularly at the contour and surface levels. He and Nakayama (1994a, 1994b) also suggested that apparent motion is perceived at the surface representation stage. Moreover, the present findings suggest that coarse object representation comprises images with low-spatial-frequency dominance. It was noted that apparent motion perception had limited capacity for attentional-processing load (Dawson, 1991). The present findings suggest that the internal representations containing incomplete depth information with low-spatialfrequency dominance allow the visual systems to reduce the processing load, relative to the fine representations close to real images. The present research demonstrates that visual systems adopt an adaptive manner for providing our percept.

\section{AUTHOR NOTE}

This research was supported by a Research Fellowship of the Japan Society for the Promotion of Science for Young Scientists to S.H. (No. 19004400) and Y.K. (No. 19005654), and by a Grant-in-Aid for Scientific Research to J.G. (Nos. 18330151 and 19001004). We are grateful to Timothy Hubbard, Greta Munger, and Alison B. Sekuler for their helpful comments and suggestions for earlier versions of the manuscript. Correspondence concerning this article should be addressed to S. Hidaka, Department of Psychology, Graduate School of Arts and Letters, Tohoku University, 27-1, Kawauchi, Aoba-ku, Sendai 980-8576, Japan (e-mail: hidaka@sal.tohoku.ac.jp).

Note-Accepted by the previous editorial team, when Thomas H. Carr was Editor.

\section{REFERENCES}

Atteneave, F., \& Block, G. (1973). Apparent motion in tridimensional space. Perception \& Psychophysics, 13, 301-307.

Baylis, G. C., \& Driver, J. (1995). Obligatory edge assignment in vision: The role of figure and part segmentation in symmetry detection. Journal of Experimental Psychology: Human Perception \& Performance, 21, 1323-1342.

Bertamini, M. (2001). The importance of being convex: An advantage for convexity when judging position. Perception, 30, 1295-1310.

Bex, P. J., Edgar, G. K., \& Smith, A. T. (1995). Sharpening of drifting, blurred images. Vision Research, 35, 2539-2546.

Brainard, D. H. (1997). The Psychophysics Toolbox. Spatial Vision, 10, 433-436.

Brehaut, J. C., \& Tipper, S. P. (1996). Representational momentum and memory for luminance. Journal of Experimental Psychology: Human Perception \& Performance, 22, 480-501.

Burr, D. (1980). Motion smear. Nature, 284, 164-165.

Burr, D., \& Ross, J. (1986). Visual processing of motion. Trends in Neurosciences, 9, 304-307.

Burt, P., \& Sperling, G. (1981). Time, distance, and feature trade-offs in visual apparent motion. Psychological Review, 88, 171-195.

Dawson, M. R. W. (1991). The how and why of what went where in apparent motion: Modeling solutions to the motion correspondence problem. Psychological Review, 98, 569-603.

Driver, J., \& BAYlis, G. C. (1995). One-sided edge assignment in vision: 2. Part decomposition, shape description, and attention to objects. Current Directions in Psychological Science, 4, 201-206.

Foster, D. H. (1975). Visual apparent motion and some preferred paths in the rotation group $\mathrm{SO}(3)$. Biological Cybernetics, 18, 81-89.

Foster, D. H. (1978). Visual apparent motion and the calculus of variations. In E. L. J. Leeuwenberg \& H. F. J. M. Buffart (Eds.), Formal theories of visual perception (pp. 67-82). Chichester, U.K.: Wiley.

Freyd, J. J., \& Finke, R. A. (1984). Representational momentum. Journal of Experimental Psychology: Learning, Memory, \& Cognition, 10, 126-132. 
Freyd, J. J., \& Finke, R. A. (1985). A velocity effect for representational momentum. Bulletin of the Psychonomic Society, 23, 443-446.

Fu, Y. X., Shen, Y., \& DAN, Y. (2001). Motion-induced perceptual extrapolation of blurred visual targets. Journal of Neuroscience, 21, 1-5.

GreEn, M. (1986). What determines correspondence strength in apparent motion? Vision Research, 26, 599-607.

Green, M., \& Odom, J. V. (1986). Correspondence matching in apparent motion: Evidence for three-dimensional spatial representation. Science, 233, 1427-1429.

Halpern, A. R., \& Kelly, M. H. (1993). Memory biases in left versus right implied motion. Journal of Experimental Psychology: Learning, Memory, \& Cognition, 19, 471-484.

He, Z. H., \& Nakayama, K. (1994a). Apparent motion determined by surface layout, not by disparity or three-dimensional distance. Nature, 367, 173-175.

He, Z. H., \& NaKayama, K. (1994b). Perceived surface shape not features determines correspondence strength in apparent motion. Vision Research, 34, 2125-2135.

Hidaka, S., Kawachi, Y., \& Gyoba, J. (2008). Depth representation of moving 3-D objects in apparent-motion path. Perception, 37, 688-703.

HubBaRd, T. L. (1995). Environmental invariants in the representation of motion: Implied dynamics and representational momentum, gravity, friction, and centripetal force. Psychonomic Bulletin \& Review, 2, 322-338.

HubBaRd, T. L. (2005). Representational momentum and related displacements in spatial memory: A review of the findings. Psychonomic Bulletin \& Review, 12, 822-851.

Hubbard, T. L., \& Bharucha, J. J. (1988). Judged displacement in apparent vertical and horizontal motion. Perception \& Psychophysics, 44, 211-221.

Kawabe, T., \& Miura, K. (2003). Figure-ground assignment based on shading. Japanese Journal of Psychonomic Science, 22, 10-17.

Kelly, M. H., \& FreYD, J. J. (1987). Explorations of representational momentum. Cognitive Psychology, 19, 369-401.

KLefFner, D. A., \& Ramachandran, V. S. (1992). On the perception of shape from shading. Perception \& Psychophysics, 52, 18-36.

Kolers, P. A. (1972). Aspects of motion perception. Oxford: Pergamon.

Kolers, P. A., \& VON GrÜNAU, M. (1976). Shape and color in apparent motion. Vision Research, 16, 329-335.

Koriat, A. (1994). Object-based apparent motion. Perception \& Psychophysics, 56, 392-404

Kourtzi, Z., \& Shiffrar, M. (1997). One-shot view invariance in a moving world. Psychological Science, 8, 461-466.

Kourtzi, Z., \& Shiffrar, M. (1999). The visual representation of threedimensional, rotating objects. Acta Psychologica, 102, 265-292.

LiU, B., \& TodD, J. T. (2004). Perceptual biases in the interpretation of 3D shape from shading. Vision Research, 44, 2135-2145.

LiU, T., Slotnick, S. D., \& Yantis, S. (2004). Human MT+ mediates perceptual filling-in during apparent motion. NeuroImage, 21, 17721780 .

MARR, D. (1982). Vision: A computational investigation into the human representation and processing of visual information. San Francisco: Freeman.

Moore, C. M., Mordkoff, J. T., \& Enns, J. T. (2007). The path of least persistence: Object status mediates visual updating. Vision Research, 47, 1624-1630.

Munger, M. P., \& Minchew, J. H. (2002). Parallels between remembering and predicting an object's location. Visual Cognition, 9, 177-194.
Munger, M. P., \& Owens, T. R. (2004). Representational momentum and the flash-lag effect. Visual Cognition, 11, 81-103.

Munger, M. P., Solberg, J. L., Horrocks, K. K., \& Preston, A. S. (1999). Representational momentum for rotations in depth: Effects of shading and axis. Journal of Experimental Psychology: Learning, Memory, \& Cognition, 25, 157-171.

Mutch, K., Smith, I. M., \& Yonas, A. (1983). The effect of twodimensional and three-dimensional distance on apparent motion. Perception, 12, 305-312.

Pelli, D. G. (1997). The VideoToolbox software for visual psychophysics: Transforming numbers into movies. Spatial Vision, 10, 437-442.

RamachandRan, V. S. (1988). Perceiving the shape from shading. Scientific American, 259, 76-83.

Ramachandran, V. S., Ginsburg, A. P., \& Anstis, S. M. (1983). Low spatial frequencies dominate apparent motion. Perception, 12, 457461.

Ramachandran, V. S., Rao, V. M., \& Vidyasagar, T. R. (1974). Sharpness constancy during movement perception. Perception, 3, 97-98.

Robins, C., \& ShePard, R. N. (1977). Spatio-temporal probing of apparent rotational movement. Perception \& Psychophysics, 22, 12-18.

RYAN, T. A. (1960). Significance tests for multiple comparison of proportion, variance, and other statistics. Psychological Bulletin, 57, 318-328.

SCHOLL, B. J. (2001). Spatiotemporal priority and object identity. Cahiers de Psychologie Cognitive, 20, 359-371.

Sekuler, A. B., \& Bennett, P. J. (1996). Spatial phase differences can drive apparent motion. Perception \& Psychophysics, 58, 174-190.

ShePARD, R. N., \& JUdD, S. A. (1976). Perceptual illusion of rotation of three-dimensional objects. Science, 191, 952-954.

ShePard, R. N., \& Zare, S. L. (1983). Path-guided apparent motion. Science, 220, 632-634.

Shioiri, S., Cavanagh, P., Miyamoto, T., \& Yaguchi, H. (2000). Tracking the apparent location of targets in interpolated motion. $\mathrm{Vi}$ sion Research, 40, 1365-1376.

Shirai, N., \& Yamaguchi, M. K. (2004). Asymmetry in the perception of motion-in-depth. Vision Research, 44, 1003-1011.

Thompson, P., Brooks, K., \& Hammett, S. T. (2006). Speed can go up as well as down at low contrast: Implications for models of motion perception. Vision Research, 46, 782-786.

Tse, P. U., \& Logothetis, N. K. (2002). The duration of 3-D form analysis in transformational apparent motion. Perception \& Psychophysics, 64, 244-265.

Ullman, S. (1978). Two dimensionality of the correspondence process in apparent motion. Perception, 7, 683-693.

Ullman, S. (1979). The interpretation of visual motion. Cambridge, MA: MIT Press.

Watson, A. B. (1986). Apparent motion occurs only between similar spatial frequencies. Vision Research, 26, 1727-1730.

Wertheimer, M. (1912). Experimentelle Studien über das Sehen von Bewegung. Zeitschrift für Psychologie, 61, 161-165.

YANTIS, S., \& NAKAMA, T. (1998). Visual interactions in the path of apparent motion. Nature Neuroscience, 1, 508-512.

Zhuo, Y., Zhou, T. G., Rao, H. Y., Wang, J. J., Meng, M., Chen, M., ET AL. (2003). Contributions of the visual ventral pathway to longrange apparent motion. Science, 299, 417-420.

(Manuscript received June 11, 2007; revision accepted for publication March 23, 2009.) 